Nota científica

\title{
Registro de Puma yagouaroundi en la Reserva de la Biosfera Zicuirán-Infiernillo, Michoacán
}

\author{
Record of Puma yagouaroundi from Zicuirán-Infiernillo Biosphere Reserve, Michoacán \\ Lina Adonay Urrea-Galeano ${ }^{\mathrm{a}, \mathrm{b}, *}$, Misael Rojas-López ${ }^{\mathrm{c}}$, Lorenzo Sánchez-Sánchez ${ }^{\mathrm{c}}$ \\ y Guillermo Ibarra-Manríquez ${ }^{\mathrm{a}}$ \\ ${ }^{a}$ Instituto de Investigaciones en Ecosistemas y Sustentabilidad, Universidad Nacional Autónoma de México, Antigua Carretera a Pátzcuaro 8701, Exhacienda de \\ San José de La Huerta, 58190, Morelia, Michoacán, México

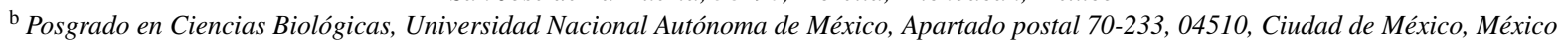 \\ ${ }^{\mathrm{c}}$ Ejido Llano Ojo de Agua, Calle Sonora s/n, Colonia Guadalupe, 61080, Churumuco, Michoacán, México \\ Recibido el 3 de junio de 2015; aceptado el 11 de diciembre de 2015 \\ Disponible en Internet el 24 de mayo de 2016
}

\section{Resumen}

Se presenta el registro fotográfico de Puma yagouaroundi (yaguarundí) en el área de amortiguamiento de la Reserva de la Biosfera ZicuiránInfiernillo. Las fotografías fueron tomadas con cámaras trampa instaladas de octubre de 2013 a octubre de 2014. Con esta evidencia se documenta la presencia de esta especie en la parte baja de la depresión del Balsas, y se sugiere que su distribución a lo largo de la sierra Madre del Sur y la zona costera de Michoacán podría tener conectividad hacia esta zona.

Derechos Reservados (C) 2016 Universidad Nacional Autónoma de México, Instituto de Biología. Este es un artículo de acceso abierto distribuido bajo los términos de la Licencia Creative Commons CC BY-NC-ND 4.0.

Palabras clave: Bosque tropical caducifolio; Cámaras trampa; Churumuco; Depresión del Balsas

\begin{abstract}
We present the record of Puma yagouaroundi (jaguarundi) with digital pictures in the buffer zone of Zicuirán-Infiernillo Biosphere Reserve. Using camera traps, photographs of Puma yagouaroundi from October 2013 to October 2014 were obtained. With this evidence, the presence of the species in the lower Balsas River basin is documented, it suggests as well that its distribution throughout the Sierra Madre del Sur and the coastal area of Michoacán could have connectivity toward this zone.

All Rights Reserved (C 2016 Universidad Nacional Autónoma de México, Instituto de Biología. This is an open access item distributed under the Creative Commons CC License BY-NC-ND 4.0.
\end{abstract}

Keywords: Tropical dry forest; Camera trap; Churumuco; Balsas River basin

Los mamíferos de gran tamaño se han convertido en especies bandera para la conservación, ya que al ser más vulnerables ante cambios drásticos en los ecosistemas, sus poblaciones se ven fuertemente reducidas (Cardillo et al., 2005), impidiendo que su presencia sea fácilmente registrada por el hombre. En

\footnotetext{
* Autor para correspondencia.

Correo electrónico: laurrea@cieco.unam.mx (L.A. Urrea-Galeano).

La revisión por pares es responsabilidad de la Universidad Nacional Autónoma de México.
}

el caso concreto de los felinos silvestres, la monitorización de sus poblaciones se ha realizado por la presencia de excretas (Lozano, Virgós, Malo, Huertas y Casanovas, 2003), el uso de radiotransmisores (Monterroso, Brito, Ferreras y Alves, 2009) y, recientemente, por medio de cámaras trampa (Charre-Medellín, Monterrubio-Rico y Guido-Lemus, 2014; Corona et al., 2014; Monroy-Vilchis, Sánchez, Aguilera-Reyes, Suárez y Urios, 2008). Una especie de felino, conocida comúnmente como yaguarundí, ha sido documentada recientemente para el estado de Michoacán (Monterrubio-Rico et al., 2012); no obstante, su 
registro en la parte baja de la depresión del Balsas no ha sido confirmado.

El yaguarundí (Puma yagouaroundi) es un félido de pequeño a mediano tamaño, tiene el cuerpo alargado con extremidades proporcionalmente cortas en relación con este, cola larga, cabeza y orejas pequeñas, estas últimas redondas; la coloración de su pelaje es generalmente uniforme en todo el cuerpo, y suele presentar 2 fases de color, una negro-grisácea -fase oscura- y otra pardo-rojiza -fase clara- (Oliveira, 1998). La distribución geográfica del yaguarundí es neotropical, distribuyéndose desde el sur de Texas hasta el sur de Brasil, Paraguay y Argentina (Emmons y Feer, 1997). En México, su área de distribución histórica abarca, por la vertiente del Pacífico, desde Sonora hasta Chiapas, y por la vertiente del Atlántico, desde Tamaulipas hasta la península de Yucatán; su presencia se ha registrado en diferentes tipos de vegetación (Aranda, 2005), desde xérica -por ejemplo, matorral xerófilo- hasta muy húmeda -como la selva alta perennifolia-. Este felino se alimenta principalmente de mamíferos pequeños -roedores- e incluye también en su dieta aves, reptiles, peces, insectos y vegetales (Aranda, 2005; Guerrero, Badii, Zalapa y Flores, 2002). Debido a la fragmentación del hábitat y las actividades humanas, el yaguarundí se considera en la República Mexicana como una especie amenazada (Semarnat, 2010). El yaguarundí, sumado a 5 especies más de felinos (Panthera onca, Puma concolor, Leopardus pardalis, Leopardus wiedii y Lynx rufus), habita en el bosque tropical caducifolio de México; la presencia de 4 de ellos ha sido documentada recientemente en la Reserva de la Biosfera Zicuirán-Infiernillo (RBZI) (Charre-Medellín et al., 2014; Corona et al., 2014). Sin embargo, el registro de $P$. yagouaroundi es anecdótico para este tipo de vegetación en la reserva. Corona et al. (2014) indican que la presencia de este felino se ha documentado con base en individuos muertos, avistamientos hechos por habitantes del sector, o bien, a partir de fotografías no publicadas.

El presente trabajo se realizó en la localidad de El Zipimo (18 42’23” N, 101 39’06.0” O), ubicada dentro del área de amortiguamiento de la RBZI; específicamente, en el polígono 4 de la subzona de preservación «Conservación comunitaria» del ejido Llano Ojo de Agua, en el municipio de Churumuco, Michoacán, a una altitud de $550 \mathrm{~m}$ snm. La extensión de la reserva es de aproximadamente 265,000 ha (Semarnat, 2007). La Estación Meteorológica de Churumuco (García, 2004) indica que en la zona de estudio se presenta un clima BSo(h')w(w)(i')g, es decir, seco, con la temperatura media anual mayor de $22^{\circ} \mathrm{C}$ y la del mes más frío mayor de $18^{\circ} \mathrm{C}$, con régimen de lluvias de verano, isotermal e intertropical; en particular, la temperatura promedio anual es de $29.5^{\circ} \mathrm{C}$ y la precipitación total anual de $564 \mathrm{~mm}$ (SMN, 2010). El periodo de lluvias se presenta generalmente de junio a septiembre, seguido de una temporada de extrema sequía, con lluvias imprevisibles.

La RBZI es una de las zonas de mayor riqueza biológica del estado de Michoacán (Conanp, 2007; Semarnat, 2014), y varias especies nativas de flora y fauna registradas en la reserva se encuentran incluidas dentro de alguna categoría de riesgo, de acuerdo con la Norma Oficial Mexicana NOM-059-Semarnat-2010 (Semarnat, 2010). El tipo de vegetación predominante en la reserva es el bosque tropical caducifolio, el cual se considera importante por la composición y la diversidad de su flora (Méndez-Toribio, MartínezCruz, Cortés-Flores, Rendón-Sandoval e Ibarra-Manríquez, 2014; Rodríguez-Jiménez, Fernández-Nava, Arreguín-Sánchez y Rodríguez-Jiménez, 2005), así como también por su papel en la conservación y recuperación de felinos (Núñez, 2007).

Durante el estudio de la dispersión de semillas de Heteroflorum sclerocarpum M. Sousa (Fabaceae), en el bosque tropical caducifolio del ejido Llano Ojo de Agua (Urrea-Galeano, 2015), se usó la técnica de fototrampeo para cuantificar la remoción de sus frutos y/o semillas por mamíferos terrestres. Para ello, en un área de $2,525 \mathrm{~m}^{2}$ se instalaron 12 cámaras trampa Bushnell Trophy. De estas, 6 fueron ubicadas en dirección al camino potencial de un mamífero y el resto fueron dirigidas exclusivamente a las parcelas experimentales de remoción. Todas las cámaras fueron colocadas a $1.5 \mathrm{~m}$ del suelo, la mitad sobre el tronco de un individuo de $H$. sclerocarpum y las restantes sobre el tronco de un árbol vecino al individuo de $H$. sclerocarpum muestreado. En ninguna de las parcelas de remoción se utilizó ningún tipo de cebo para atraer animales. Las cámaras trampa estuvieron activas durante $24 \mathrm{~h}$, desde octubre de 2013 hasta octubre de 2014. La revisión de las fotografías captadas se realizó mensualmente.

Se obtuvieron 3 fotografías consecutivas de un mismo individuo de P. yagouaroundi (fig. 1). Esta especie se registró el 24 de junio de 2014, a las $17 \mathrm{~h}$, por lo que representa una evidencia de la presencia de esta especie en la RBZI. Adicionalmente se registraron las siguientes especies: Bassariscus astutus (cacomixtle), Dasypus novemcinctus (armadillo), Didelphis virginiana (tlacuache), L. pardalis (ocelote), Nasua narica (tejón), Odocoileus virginianus (venado de cola blanca), P. onca (jaguar), Pecari tajacu (pecarí de collar), Spermophilus adocetus (cuinique), Spilogale pygmaea (zorrillo), Tamandua mexicana (oso hormiguero) y Urocyon cinereoargenteus (zorra gris). De las especies previamente mencionadas, $P$. onca (fig. 2A) y $T$. mexicana (fig. 2B) son consideradas en la categoría «en peligro de extinción» (Semarnat, 2010).

La hora en que se fotografió al individuo de $P$. yagouaroundi evidencia que la especie presenta actividad diurna, información que es congruente con la registrada en otras investigaciones (Aranda, 2005; Botello et al., 2013; Farías et al., 2015; Oliveira, 1998), y, además, consistente con el registro hecho para esta especie en otras regiones del estado de Michoacán (Monterrubio-Rico et al., 2012). El mes en que se tomó la fotografía corresponde al inicio de la época de lluvias, cuando los árboles ya han comenzado con la producción de hojas y, por lo tanto, la cobertura de la vegetación se vuelve densa, condición que se ha considerado necesaria para la conservación de esta y otras especies de felinos (Núñez, 2007). El tipo de vegetación en la cual se registró el individuo concuerda con el reportado en otras investigaciones hechas en diferentes estados de México (Botello et al., 2013; Farías et al., 2015; Guerrero et al., 2002; Monterrubio-Rico et al., 2012). El sitio donde se tomó la fotografía se encuentra aproximadamente a $4.5 \mathrm{~km}$-distancia en línea recta usando el mapa del área de estudio que despliega el programa Google Earth-, al norte de la zona habitada del 


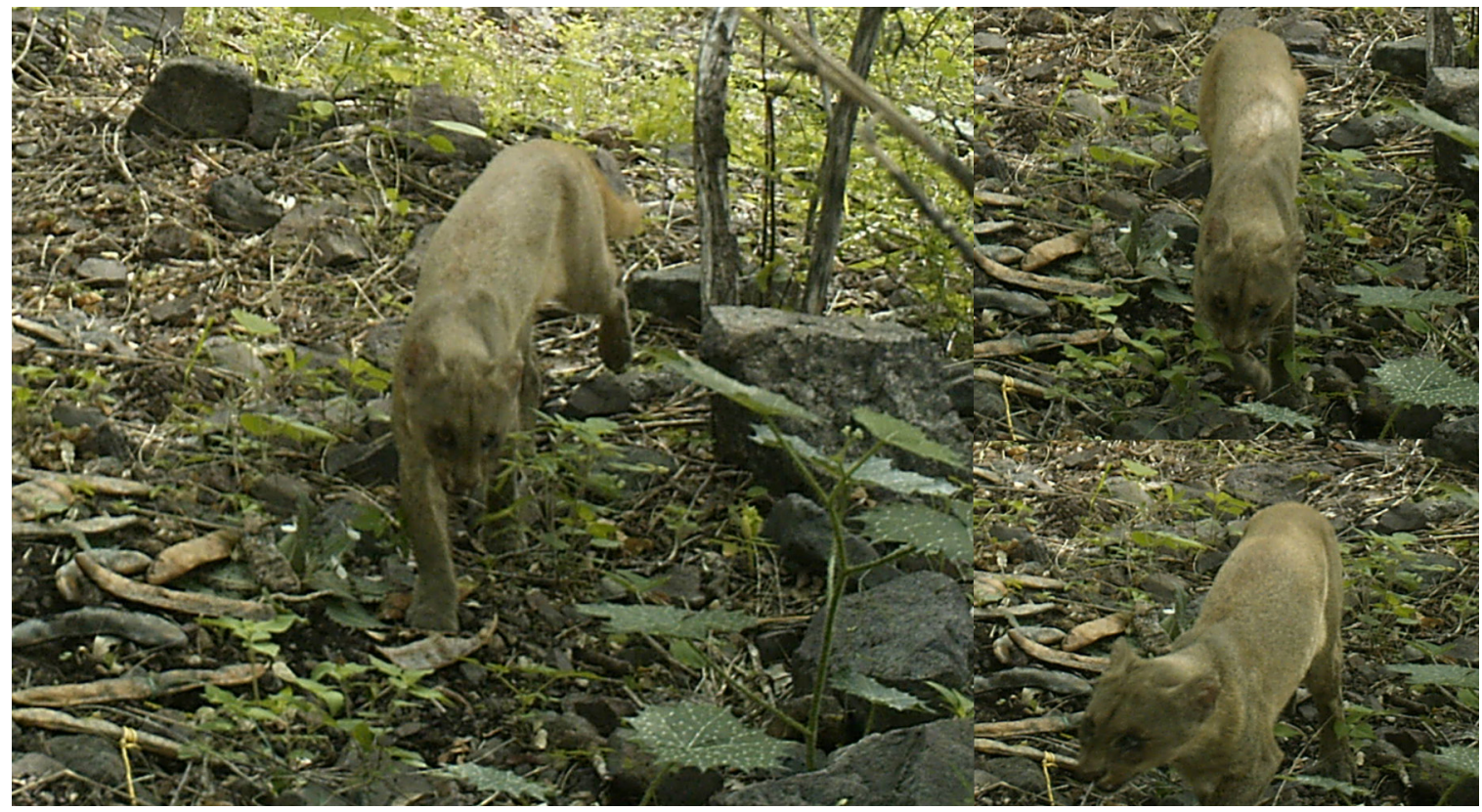

Figura 1. Individuo de Puma yagouaroundi fotografiado en el área de amortiguamiento de la Reserva de la Biosfera Zicuirán-Infiernillo, en el municipio de Churumuco, Michoacán.

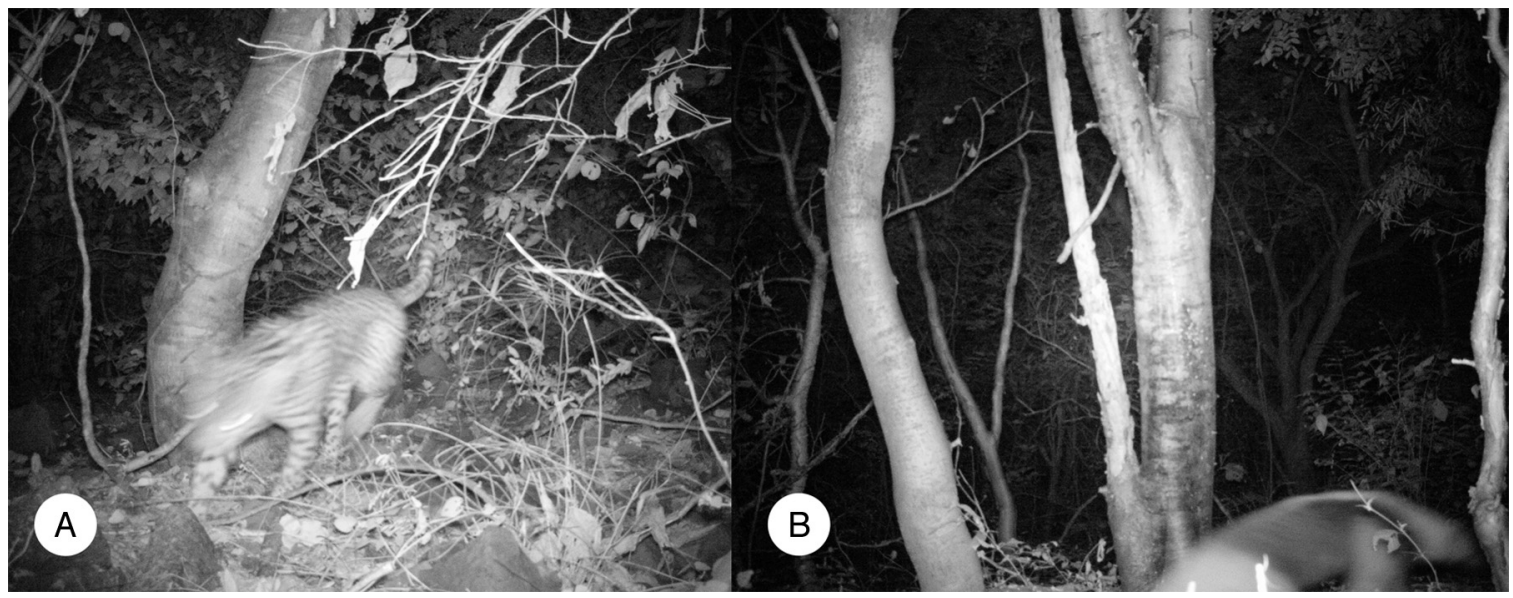

Figura 2. Fotografías de 2 especies de mamíferos terrestres en peligro de extinción, registradas en el área de amortiguamiento de la Reserva de la Biosfera Zicuirán-Infiernillo, en el municipio de Churumuco, Michoacán. A) Panthera onca. B) Tamandua mexicana.

ejido Llano Ojo de Agua, en un área cercana a un cauce temporal de agua. Aparentemente los pobladores del ejido no están muy familiarizados con $P$. yagouaroundi, pues incluso lo han confundido con el puma ( $P$. concolor), pero esto demanda una evaluación futura más detallada.

El registro de $P$. yagouaroundi en el área de amortiguamiento de la RBZI sugiere que la distribución planteada para esta especie por Monterrubio-Rico et al. (2012) a lo largo de la sierra Madre del Sur y la zona costera de Michoacán podría tener conectividad hacia la depresión del Balsas. Adicionalmente, este registro indica la importancia de seguir conservando los ecosistemas donde habitan los felinos silvestres, particularmente del bosque tropical caducifolio, uno de los ecosistemas más amenazados a nivel mundial (Olson y Dinerstein, 2002). Es por ello que el propósito fundamental de esta contribución es documentar la presencia de esta especie y resaltar el registro de $P$. onca y
T. mexicana (fig. 2). De esta manera podrían priorizarse recursos económicos hacia las áreas de conservación comunitaria de los ejidos donde se encuentra la reserva, mediante la estrategia de pago por servicios ambientales o proyectos para la monitorización de especies de fauna y flora amenazadas. Apoyos que faciliten el establecimiento de fuentes de ingreso económico a mediano plazo seguramente podrían incidir en la conformación de estrategias que garanticen el mantenimiento a largo plazo de las comunidades vegetales que habitan las especies de mamíferos mencionadas en el presente estudio, y de la biota con la que cohabitan.

Agradecemos a los investigadores Rodrigo Núñez y Eduardo Mendoza por la determinación de los felinos; a los habitantes del ejido Llano Ojo de Agua por facilitarnos el acceso a sus terrenos y brindarnos su constante acompañamiento en campo. Especialmente a la Dra. Ellen Andresen y a la Fundación Idea Wild, por 
facilitar equipo para el trabajo en campo. De igual manera, se reconoce la lectura crítica del documento por la bióloga Rosamond Coates. También agradecemos a los 2 revisores anónimos sus pertinentes contribuciones al manuscrito. Este estudio forma parte del proyecto de investigación de maestría de la primera autora, dentro del Posgrado en Ciencias Biológicas, de la Universidad Nacional Autónoma de México, el cual fue apoyado por una beca del Consejo Nacional de Ciencia y Tecnología.

\section{Referencias}

Aranda, M. (2005). Herpailurus yagouaroundi. En G. Ceballos y G. Oliva (Eds.), Los mamíferos silvestres de México (pp. 358-359). Ciudad de México: Fondo de Cultura Económica y Comisión Nacional para el Conocimiento y Uso de la Biodiversidad.

Botello, F., Villaseñor, E., Guevara, L., Méndez, A., Cortés, A., Iglesias, J., et al. (2013). Registros notables del zorrillo manchado (Spilogale angustifrons) y del jaguarundi (Puma yagouaroundi) en la Reserva de la Biosfera de Tehuacán-Cuicatlán, Oaxaca, México. Revista Mexicana de Biodiversidad, 84, 713-717.

Cardillo, M., Mace, G., Jones, K., Bielby, J., Bininda-Emonds, O., Sechrest, W., et al. (2005). Multiple causes of high extinction risk in large mammal species. Science, 309, 1239-1241.

Charre-Medellín, J. F., Monterrubio-Rico, T. C. y Guido-Lemus, D. (2014). Nuevo registro de jaguar (Panthera onca), en el centro occidente de México. Revista Mexicana de Biodiversidad, 85, 1295-1299.

Conanp (Comisión Nacional de Áreas Naturales Protegidas). (2007). Memoria de la consulta pública de la Reserva de la Biosfera Zicuirán-Infiernillo, en el estado de Michoacán. Ciudad de México: Conanp.

Corona, E., Núñez, R., Anguiano, D., Tornez, M., Solorio, I. y Torres, A. (2014). Zicuiran-Infiernillo Biosphere Reserve, a refuge for wild cats in Tierra Caliente, Michoacan, Mexico. The Wild Felid Monitor, 7, 16-17.

Emmons, L. y Feer, F. (1997). Neotropical rainforest mammals, a field guide. Illinois, Chicago: The University of Chicago Press.

Farías, V., Téllez, O., Botello, F., Hernández, O., Berruecos, J., Olivares, S. J., et al. (2015). Primeros registros de 4 especies de felinos en el sur de Puebla, México. Revista Mexicana de Biodiversidad, 86, 1065-1071.

García, E. (2004). Modificaciones al sistema de clasificación climática de Köppen (quinta edición). Ciudad de México: Instituto de Geografía, Universidad Nacional Autónoma de México.

Guerrero, S., Badii, M. H., Zalapa, S. S. y Flores, A. E. (2002). Dieta y nicho de alimentación del coyote, zorra gris, mapache y jaguarundi en un bosque tropical caducifolio de la Costa del Sur del estado de Jalisco, México. Acta Zoológica Mexicana, 86, 119-137.

Lozano, J., Virgós, E., Malo, A., Huertas, D. y Casanovas, J. (2003). Importance of scrub-pastureland mosaics for wild-living cats occurrence in a Mediterranean area: Implications for the conservation of the wildcat (Felis silvestris). Biodiversity and Conservation, 12, 921-935.
Méndez-Toribio, M., Martínez-Cruz, J., Cortés-Flores, J., Rendón-Sandoval, F. J. y Ibarra-Manríquez, G. (2014). Composición, estructura y diversidad de la comunidad arbórea del bosque tropical caducifolio en Tziritzícuaro, Depresión del Balsas, Michoacán, México. Revista Mexicana de Biodiversidad, $85,1117-1128$.

Monroy-Vilchis, O., Sánchez, O., Aguilera-Reyes, U., Suárez, P. y Urios, V. (2008). Jaguar (Panthera onca) in the state of Mexico. The Southwestern Naturalist, 53, 533-537.

Monterroso, P., Brito, J., Ferreras, P. y Alves, P. (2009). Spatial ecology of the European wildcat in a Mediterranean ecosystem: dealing with small radio-tracking datasets in species conservation. Journal of Zoology, 279, $27-35$.

Monterrubio-Rico, T., Charre-Medellín, J., Zavala-Paramo, M., Cano-Camacho, H., Pureco-Rivera, M. y León-Paniagua, L. (2012). Evidencias fotográfica, biológica y genética de la presencia actual de jaguaroundi (Puma yagouaroundi) en Michoacán, México. Revista Mexicana de Biodiversidad, 83, $825-833$.

Núñez, R. (2007). Distribución y situación del jaguar en el occidente de México. En C. Ceballos, R. Chávez, R. List, y H. Zarza (Eds.), Conservación y manejo del jaguar en México: estudios de caso y perspectivas (pp. 25-40). Ciudad de México: Conabio-Alianza WWF/Telcel-Universidad Nacional Autónoma de México.

Oliveira, T. G. (1998). Herpailurus yagouaroundi. Mammalian Species, 578, $1-6$.

Olson, D. y Dinerstein, E. (2002). The global 2000: priority ecoregions for global conservation. Annals of the Missouri Botanical Garden, 89, 199-224.

Rodríguez-Jiménez, C., Fernández-Nava, R., Arreguín-Sánchez, M. L. y Rodríguez-Jiménez, A. (2005). Plantas vasculares endémicas de la cuenca del río Balsas, México. Polibotánica, 20, 73-99.

Semarnat (Secretaría de Medio Ambiente y Recursos Naturales). (2007). Programa de manejo del Área Natural Protegida con la categoría de Reserva de la Biosfera Zicuirán-Infiernillo. Diario Oficial de la Federación. 30 de noviembre de 2007. México.

Semarnat (Secretaría de Medio Ambiente y Recursos Naturales). (2010). Norma Oficial Mexicana NOM-059-SEMARNAT-2010, Protección ambientalEspecies nativas de México de flora y fauna silvestres-Categorías de riesgo y especificaciones para su inclusión, exclusión o cambio-Lista de especies en riesgo. Diario Oficial de la Federación. 30 de diciembre de 2010. México.

Semarnat (Secretaría de Medio Ambiente y Recursos Naturales). (2014). Programa de manejo Reserva de la Biosfera Zicuirán-Infiernillo. Ciudad de México: Semarnat.

SMN (Servicio Meteorológico Nacional). (2010). Normales climatológicas de la estación de Churumuco durante el periodo de 1981 al 2010. Recuperado el 30 de marzo del 2016 de: http://smn.cna.gob.mx/index.php?option= com_content $\&$ view $=$ article $\&$ id $=42 \&$ Itemid $=75$

Urrea-Galeano, L. A. (2015). Dispersión de semillas de Heteroflorum sclerocarpum M. Sousa (Fabaceae) en un bosque tropical caducifolio en la Depresión del Balsas, Michoacán [tesis de maestría]. Posgrado en Ciencias Biológicas (Ecología). Universidad Nacional Autónoma de México. México, D.F. 